\title{
Nanoscale Chemical Imaging via AFM coupled IR Spectroscopy
}

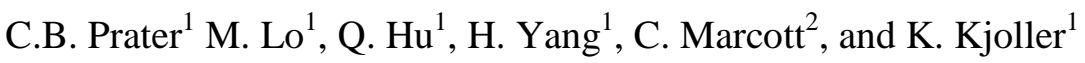 \\ ${ }^{1}$ Anasys Instruments, Santa Barbara, California 93101 USA \\ ${ }^{2}$ Light Light Solutions, Athens, Georgia 30608 USA
}

Conventional infrared spectroscopy is one of the most widely used tools in science and industry to identify materials via vibrational resonances of chemical bonds, but optical diffraction limits its spatial resolution to the scale of many microns. Atomic force microscopy (AFM) enjoys excellent spatial resolution and can measure mechanical, electrical, magnetic and thermal properties of materials, but has historically lacked the ability to perform robust chemical analysis. Two techniques, (1) AFM-based infrared spectroscopy (AFM-IR) and (2) scattering scanning near field optical microscopy (s-SNOM) have been developed which couple AFM with an IR source allowing the chemical identification capabilities of IR spectroscopy to extend to the nanoscale. As complementary techniques, AFM-IR and s-SNOM together provide an unrivaled capability to perform nanoscale chemical analysis on a diverse range of organic, inorganic, photonic and electronic materials.

The AFM-IR technique achieves nanoscale spatial resolution by using the AFM probe as a local sensor of the IR absorption [1]. A sample is irradiated with light from a pulsed, tunable infrared laser. When the IR laser is tuned to a wavelength where the sample has an absorption peak, a portion of the incident IR light is absorbed and converted into heat resulting in a rapid thermal expansion of the absorbing region. This generates an impulse force on the AFM tip, inducing resonant oscillations of the AFM cantilever. IR absorption spectra can then be obtained by measuring the cantilever oscillation amplitude as a function of laser wavenumber. Because the amplitude of induced cantilever oscillation is directly proportional to the IR absorption of the sample [2], absorption spectra obtained by AFM-IR compare very well to conventional FTIR without the use of modelling or reference samples. More recent extensions of this technique have allowed chemical measurements to be performed on samples as thin as individual monolayers [3]. Figure 1 shows an example application of the AFM-IR measurement, nanoscale chemical measurements of the environmental degradation of a biomedical material. One challenge to implantable devices is the possible degradation of the material due to exposure to the in vivo environment. Polyurethane was exposed to this simulated environment for sufficient time to allow the initiation of surface degradation. The chemical mechanism of this degradation was then analyzed and mapped using the AFM-IR technique. The AFM-IR spectra clearly show the chain scission which occurs in the ether bonds of the polyurethane by the reduction in the peak at $1108 \mathrm{~cm}^{-1}$. It also shows the formation of alcohol groups by the increase in the $1176 \mathrm{~cm}^{-1}$ absorption band and carboxylate groups (COO-) shown by the increase in the broad bands at 1652 and around $1400 \mathrm{~cm}^{-1}$. These absorption bands can be used to map the degradation and determine how the degradation initiates and extends into the sample, information that was previously not achievable at this length scale.

The s-SNOM technique has been used in the SPM field for more than two decades [4]. It uses a metallized AFM tip to enhance and scatter radiation from a nanometer scale region of the sample. The scattered radiation is detected in the far field and carries information about the complex optical properties of the nanoscale region of the sample under the metallized tip. Both the optical amplitude 
and phase of the scattered light can be measured typically using reference samples (e.g. gold or silicon) to correct the measurements for unknown phase contributions from the light source and tip. With appropriate models, these measurements can be converted into measurements of the complex optical constants $(\mathrm{n}, \mathrm{k})$ of the material under the tip. In some cases, the optical phase versus wavelength provides an approximation to a conventional absorption spectrum. As well as providing chemical imaging, the s-SNOM technique can also image electrical properties as demonstrated by the imaging of surface plasmon polaritons (SPP) in graphene [5], shown in Fig. 2. The SPPs are excited by the s-SNOM tip and then scattered or reflected by grain boundaries, edges or defects in the graphene. This generates interference which can be visualized with high spatial resolution using $\mathrm{s}-\mathrm{SNOM}$ and provides information on the electronic structure of the graphene.

\section{References:}

[1] Dazzi, A., et al., Local infrared microspectroscopy with subwavelength spatial resolution with an atomic force microscope tip used as a photothermal sensor. Opt. Lett., 2005. 30(18): p. 2388-2390.

[2] Dazzi, A., Theory of infrared nanospectroscopy by photothermal induced resonance. J. Appl. Phys., 2010. 107(12): p. 124519.

[3] Lu, F.; Belkin, M., Infrared absorption nano-spectroscopy using sample photoexpansion induced by tunable quantum cascade lasers. Opt. Exp. 2011, 19, 19946.

[4] Zenhausern, F., et al, Apertureless Near-Field Optical Microscope. Applied Physics Letters 199465 (13), p. 1623-1625.

[5] Gerber, J. A. et al., Phase-Resolved Surface Plasmon Interferometry of Graphene. Phys. Rev. Lett., 2014. 113, 055502.

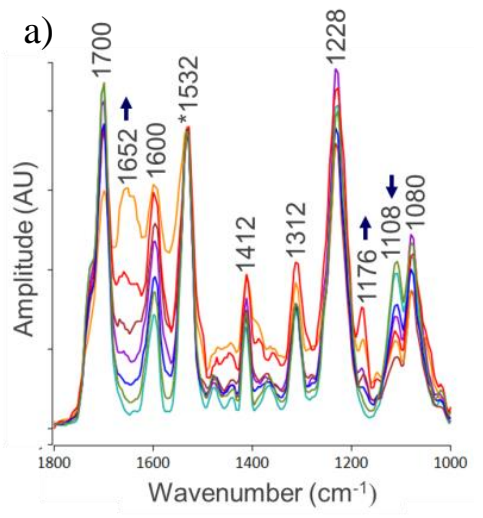

b)

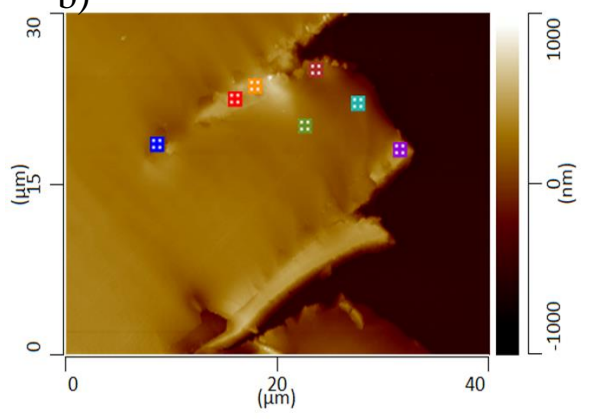

Figure 1. a) AFM-IR spectra and b) AFM topography image of a section of a degraded polyurethane sample showing the chemical variation caused by the exposure of the polyurethane to a simulated in vivo environment.

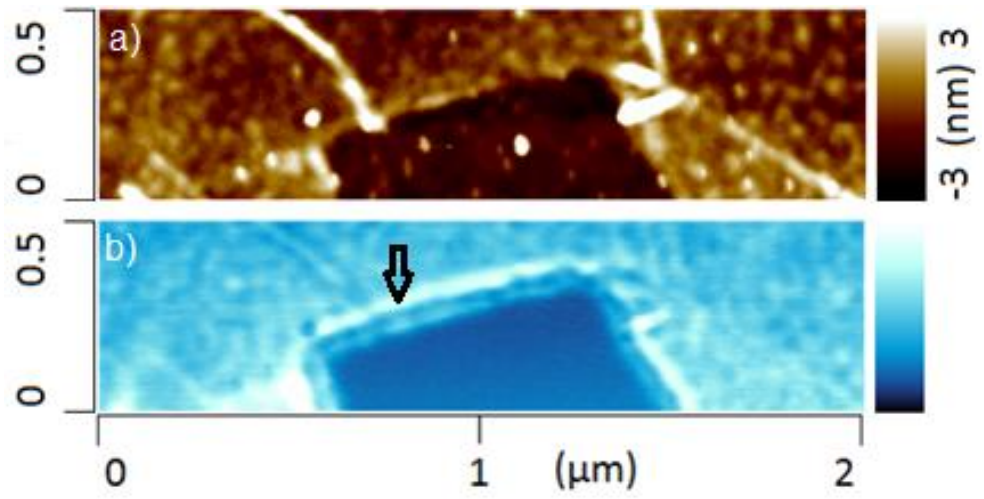

Figure 2. a) AFM topography and b) near field amplitude, collected at 925 $\mathrm{cm}^{-1}$ of a graphene sample showing the defects and grain boundaries which result in the plasmon interference in the near field image (indicated by the arrow). 